\title{
Cooperation in Underwater Sensor Networks
}

\author{
Florent Garcin, Mohammad Hossein Manshaei, and Jean-Pierre Hubaux
}

\begin{abstract}
In underwater sensor networks (UWSNs), one of the main concerns is to reduce the energy consumption in order to maximize the lifetime. This work focuses on multiple sensor networks governed by different authorities. If the authorities are willing to cooperate by forwarding each others' packets, the global lifetime could be increased. However, a selfish authority could exploit the others' collaborative behaviors. To investigate this cooperation, we apply concepts such as evolutionary game theory. A first analysis of a simple model determines that cooperation underwater can emerge without incentives. Then, we explore with an extended model what influences cooperation and in which way. Finally, we demonstrate how a new authority can fine tune its strategy when deploying its UWSN.
\end{abstract}

\section{INTRODUCTION}

Understanding the key mechanisms of the oceans is crucial for the knowledge of earth's climate and atmosphere. Although water covers most of our planet, the underwater world remains hostile to humans and is little explored. Over the past few years, there has been a relentless effort to investigate the abyssal depths of the oceans. This infatuation is highly motivated by the applications of underwater communications, such as undersea resources exploration and exploitation, and their economic implications. Underwater sensor networks have many applications. Nonetheless, underwater communications are a very challenging topic to which researchers have only recently shown a growing interest [11]. In terrestrial wireless communication, the energy to transmit a packet increases at least with the square of the distance of transmission. However in water, it increases not only with the distance but also with the frequency of transmission. Sensors usually draw power from batteries and recharging, and changing them is almost impossible in this case. Therefore, it is better from an energy standpoint to transmit packets in many small hops than a single large hop.

In a near future, we forecast cohabitation between underwater applications. For instance, an oil company uses an underwater sensor network for system parameters monitoring of an oil extraction facility. In the same vicinity, exploration vehicles of another company are deployed to find new spots. Another sensor networks may be owned by the government to monitor these activities and for other purposes such as natural disasters prevention or military surveillance. Finally, non-government organisations deploy their network as well for environmental monitoring. Thus, the case of a single

F. Garcin is with the Artificial Intelligence Laboratory (LIA), EPFL, 1015 Lausanne, Switzerland.florent.garcin@epfl.ch

M. H. Manshaei and J.-P. Hubaux are with the Laboratory for Computer Communications and Application (LCA), the School of Computer \& Communication Science, EPFL, 1015 Lausanne, Switzerland. \{hossein.manshaei, jean-pierre.hubaux\}@epfl.ch authority dictating policies to all sensor networks will soon be obsolete. Authorities can reduce energy consumption by mutual cooperation and thus increase their network lifetime. This is done by forwarding packets of other authorities. However, a selfish authority might exploit the others' cooperative behaviors.

In the research literature, cooperation among nodes is encouraged by either reputation or payment mechanisms. The former is based on the monitoring of a node's behavior by its neighbors [7], [8]. Payment schemes try to reward cooperative nodes [3], [18]. These mechanisms are mainly feedback based and thus generate additional traffic. In addition, they are prone to possible attacks. For these reasons, they are not appropriate for an underwater environment because of the propagation delay but also because of the energy consumption. Nevertheless, a common goal could be enough to encourage spontaneous cooperation.

In this paper, we show first that cooperation can emerge underwater without incentives. A simplified model demonstrates that the quality of service influences cooperation. With a more realistic model, we discuss what affects the cooperation level and in which way. This model highlights that the strategies, as well as the topology and the parameters, are related to the cooperation level. Finally, we demonstrate how a new authority can fine tune its strategy when deploying an underwater sensor network. To the best of our knowledge, cooperation in UWSNs has never been investigated.

The remainder of this paper begins with a brief review of the literature on cooperation in terrestrial environments. We introduce the key concepts of game theory and evolutionary game theory in Section III. Then, in Section IV, we present the system and communication models. Section V explores cooperation issues by analyzing a simplified model of UWSNs. The game in Section VI is an extension of the previous one and focuses on what influences cooperation and in which way. We relax the assumptions of the first two games to analyze in Section VII the strategic interactions between three and more authorities. Finally, we conclude our work in Section VIII.

\section{RELATED WORK}

Publications focusing on wireless sensor networks assume that cooperation should be fostered by incentives. Incentives are usually provided by either micro-payment or reputation schemes. Cooperation without incentives has been addressed in [2], [6] for sensor networks. We present in the following their main contributions and how our work differs from them.

In [6], Felegyhazi et al. consider sensor networks that are deployed in the same area but controlled by different 
authorities. Their model diverges from ours in several aspects. First, when a sensor runs out of battery power, the authority is dismissed and routes are recalculated. In our model, we recalculate the routes every time a sensor dies and we wait until all sensors belonging to an authority are dead before removing it. Second, the payoff is a subjective value representing the importance of a successful round, whereas in our case, the payoff is the network lifetime and is received at the end of the game. Last, when an authority has a number of received packets below a given threshold, it still has the possibility to choose actions that could lead to an unsuccessful round. We restrict this to guarantee a higher quality of service.

Buttyan et al. [2] study the cooperation in multi-domain sensor networks governed by different authorities. Their work is closely related to [6] but has slight modifications. The authors show that cooperative Nash equilibria exist and rely on smart strategies that try to exploit the others. Then, they classify the Nash equilibria into levels of cooperation. In case there are more than one NE, they select the most cooperative one. However in our models, we select all Nash equilibria.

Crosby and Pissinou [5] analyze the forwarding of packets between authorities of fixed and mobile wireless sensor networks. Their results show that, in the case of packet forwarding between mobile wireless sensor networks, the strategy of always cooperating is not evolutionary stable. However, in some scenarios, this strategy stabilizes over time. They also propose the Patient Grim Strategy that enforces cooperation by punishment and prove that it is a Nash equilibrium. Compared to our model, the moves of a player are limited to cooperation or defection regarding an opponent's packet. In our scenarios, the decision is made on both the self and the opponent's packets. They study only two strategies, whereas in our work other strategies lead to Nash equilibria with higher payoffs. The previous rounds do not influence the decision of the following round. However, we provide authorities a history to store the previous outcomes. Finally, the payoffs are subjective values. In this work, we use the network lifetime as the payoff.

\section{INTRODUCTION TO GAME THEORY AND EVOLUTIONARY GAME THEORY}

Game theory is a branch of mathematics mainly used in economics to study strategic interactions between decision makers. From the seminal work of von Neumann and Morgenstern [16], game theory has greatly evolved. It has been applied in various domains such as politics, computer science or biology. In this section, we review the key concepts of this theory, which we will use in the remaining of this work. Interested readers may refer to [10], [12] for further details or [4] for the application to wireless networks.

In this work, we will study different models of interactions and focus on non-cooperative ones. These models are called games and the decision makers are players. We describe a game by $G=\{P, S, U\} . P$ is the set of players $P=$ $\left\{p_{1}, p_{2}, \ldots, p_{N}\right\}$ and we use for simplicity $p_{-i}$ to refer to all players except $i$. Players pick up one strategy among a set of possible strategies $s_{i} \in S$. A strategy profile $s=$ $\left\{s_{1}, s_{2}, \ldots, s_{N}\right\}$ is the set of chosen strategies. Finally, each player evaluates his choice against the others through a payoff or utility function $u_{i}(s) \in U$. This function reflects the player's objective. We assume that players are rational and try to maximize their respective payoffs.

There exist two kinds of strategy. When a player takes an action with a probability 1 , it is a pure strategy. A mixed strategy assigns a probability to each pure strategy. The player will choose a pure strategy based on the distribution given by the mixed strategy.

In a game, we are usually interested in predicting the strategy of each player and find the solution. There exist many methods to do this. We use here the Nash equilibrium (NE) concept. A Nash equilibrium is a strategy profile $s^{*}=$ $\left\{s_{1}^{*}, s_{2}^{*}, \ldots, s_{N}^{*}\right\}$ such that

$$
u_{i}\left(s_{i}^{*}, s_{-i}^{*}\right) \geq u_{i}\left(s_{i}, s_{-i}^{*}\right), \forall i \text { and } \forall s_{i} \in S
$$

In other words, a NE exists when no player has incentive to deviate unilaterally from his strategy. Every game with finite number of players and finite number of pure strategies has at least one NE involving pure or mixed strategy profiles.

Once we have identified the Nash equilibria of a game, we study their efficiency. By using the concept of Pareto optimality, we compare strategy profiles to identify the best Nash equilibria in the game. A strategy profile $s$ is Paretooptimal if it is not possible to increase the payoff of any player without decreasing the payoff of another player.

Previously, we relied on the NE concept to find the solution of games in which each player uses a strategy that is the best response to the opponent's. Once the best responses are known, neither player will change their strategy. We relax this assumption to analyze the strategic interactions between three and more players, thanks to evolutionary game theory.

The evolutionary game theory (EGT) applies biology concepts to game theory and proposes to study the evolution and strategic interactions over time in a large population. The basic idea is that a group of individuals playing a given strategy will change over time in response to the strategies played by other groups. Thus, the population evolves. In the current population, individuals who play a given strategy and perform better than others will leave more offspring. In the next generation, the population will change.

The equivalent concept of Nash equilibrium in EGT is the evolutionary stable strategy (ESS) [17]. The strategy $s^{*}$ is evolutionary stable if $\forall i$ and $\forall s \neq s^{*}$

1) $u_{i}\left(s_{i}^{*}, s_{-i}^{*}\right)>u_{i}\left(s_{i}, s_{-i}^{*}\right)$, or

2) $u_{i}\left(s_{i}^{*}, s_{-i}^{*}\right)=u_{i}\left(s_{i}, s_{-i}^{*}\right)$ and $u_{i}\left(s_{i}^{*}, s_{-i}\right)>u_{i}\left(s_{i}, s_{-i}\right)$

Therefore, a population is evolutionary stable if it is resistant to invasion by a small number of mutants playing a different strategy. In other words, we have an initial population of individuals with the same strategy. We introduce then a small group of mutants with a different strategy. If the initial population's strategy is an ESS, the selection process will eventually eliminate the invaders. The mutants' strategy will change to the ESS. 
The replicator dynamic specifies how the population evolves in time. To define what a replicator dynamic is, we need first to introduce how to evaluate the population and strategies.

A population is represented by a population profile $\vec{x}=$ $\left(x_{1}, x_{2}, \ldots\right)$ assigning for each pure strategy $s_{i} \in S$ the proportion of individuals $x_{i}$ playing that strategy over the whole population. We denote by $\pi(\sigma, \vec{x})$ the payoff of the portion of individuals playing strategy $\sigma$ and we calculate it as the average payoffs of individuals playing strategy $s_{i}$ :

$$
\pi(\sigma, \vec{x})=\sum_{i} x_{i} u\left(\sigma, s_{i}\right)
$$

The average payoff for the whole population is

$$
\bar{\pi}(\vec{x})=\sum_{i} \pi\left(s_{i}, \vec{x}\right)
$$

The population is evolved by the replicator dynamic. Depending on the evolution model we use, there exist several replicator dynamics. According to Darwin's theory of evolution, the fittest individuals should survive and reproduce for the next generation, and the least fit should die off. We describe this with the discrete time dynamic function:

$$
x_{i}(t+1)=x_{i}(t) \frac{\pi\left(s_{i}, \vec{x}\right)}{\bar{\pi}(\vec{x})}
$$

One generation of the evolution process is as follows. First, we distribute randomly the strategies to the individuals with respect to the initial population profile $\vec{x}(0)$. Then, all individuals play the game. We evaluate the payoffs $\pi\left(s_{i}\right)$ and $\bar{\pi}(\vec{x}(0))$. The population profile for the next generation $\vec{x}(1)$ is updated according to the replicator dynamic and the strategies are distributed again randomly in function of the new population profile. The evolution ends when all individuals have the same strategy. We will use this technique in Section VII, where we evaluate the interactions between several sensor network authorities.

\section{System And Communication Models}

To study cooperation in underwater sensor networks, we consider three scenarios. In the first scenario (Section V), two authorities have deployed one sensor and one buoy each. The buoys act as sinks and collect sensed data. Sensors are at the depth $z$ and the distance between one sensor and the buoy is the same as the distance between the two sensors. The sensors have the same amount of battery power. Once one sensor runs out of it, it is considered as dead and removed. This scenario is depicted in Fig. 1. The time is divided in time slots. Each sensor sends a single data packet to its respective buoy in each time slot. The length of each packet is the same. The sensor has to choose between a single hop transmission and two hops, through the other sensor.

The second scenario presented in Section VI is an extension of the first but with realistic conditions. Each authority governs a network with the same number of sensors randomly spread in a pool. We explore two topologies. In the first topology (shared location), the two buoys are located at the center of the pool, whereas in the second (distinct

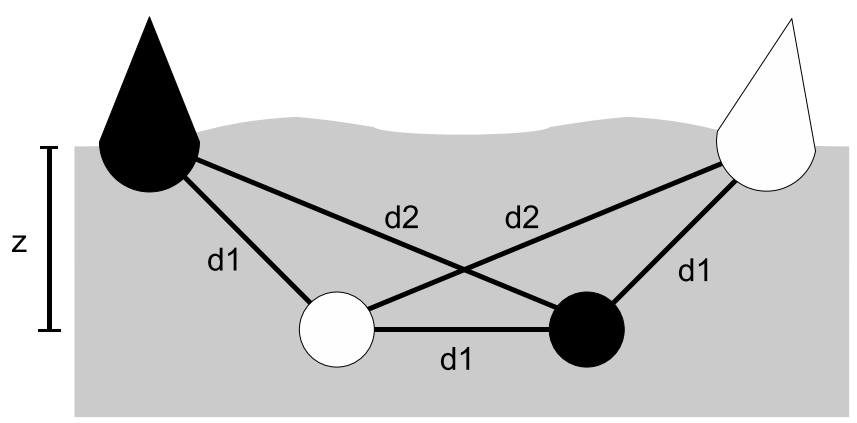

Fig. 1. A simple scenario with two authorities (black and white).

location) they are separated at equal distance from the center of the pool. Everytime a sensor runs out of battery power, we calculate the routes using the minimum energy path routing towards the buoy [1]. Every sensor has two paths: local and global. The local path contains only sensors governed by the same authority. The global path is computed with all the sensors. We remove an authority when all its sensors are dead.

The previous two scenarios have only two authorities. In the last scenario (Section VII), we relax this assumption and take more than two authorities. Moreover, we focus our study on the topology with shared buoys.

Because radio waves do not propagate well in the water, communication is done by the mean of acoustic channels and differs in many aspects. The propagation speed is very slow (around $1.5 \cdot 10^{3} \mathrm{~m} / \mathrm{s}$ ). It varies with the pressure, temperature and salinity and thus highly depends on the environment. The transmission loss is caused by spreading and absorption and is related not only to the distance between nodes but also to the frequency. In addition, the noise is not the same and comes from various sources. Finally, underwater acoustic channels are prone to multipath interference.

According to [15], the minimum signal-to-noise ratio (SNR) for a successful underwater transmission is around $22 \mathrm{~dB}$. This communication model aims at guaranteeing a SNR of $25 \mathrm{~dB}$ at the receiver. We describe it with the passive sonar equation [15]:

$$
S N R=S L-T L-N L+D I
$$

where $S L, T L, N L$ and $D I$ are, in $\mathrm{dB}$, the source level, transmission level, noise level and directivity index respectively. As we consider omnidirectional hydrophones, the directivity index is 0 . The transmission loss over a distance $d$ in meter for a signal of frequency $f$ in $\mathrm{kHz}$ is given by [15]

$$
T L=d^{k} a(f)^{d}
$$

Expressed in $\mathrm{dB}$, we have

$$
T L=k \cdot 10 \log _{10} d+\alpha(f) d \cdot 10^{-3}
$$

where $k$ is the spreading factor and $\alpha(f)$ the absorption coefficient. In the following, we assume a practical spreading factor $k=1.5$. We use Thorp's formula [14], [15] for the 


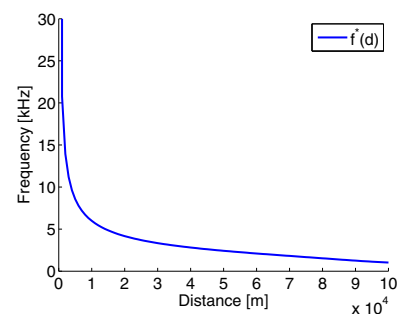

(a) Optimal frequency $f^{*}$

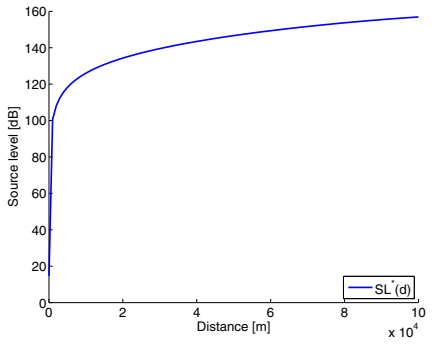

(b) Minimum source level $S L^{*}$
Fig. 2. Optimal frequency and minimum source level for a SNR of $25 \mathrm{~dB}$.

absorption coefficient in $\mathrm{dB} / \mathrm{km}$ :

$$
\alpha(f)=0.11 \frac{f^{2}}{1+f^{2}}+44 \frac{f^{2}}{4100+f^{2}}+2.75 \cdot 10^{-4} f^{2}+0.003
$$

with $f$ in $\mathrm{kHz}$.

The noise is composed of four components: turbulence, shipping, waves and thermal noise. For simplicity, we employ a useful approximation presented in [13], namely:

$$
N L=50-18 \log _{10} f
$$

with $f$ in $\mathrm{kHz}$.

From the above equations, we have the expression for the signal level

$S L=S N R+k 10 \log _{10} d+\alpha(f) d \cdot 10^{-3}+50-18 \log _{10} f$

In order to minimize the energy consumption, we obtain the minimum source level by taking the derivative and using (3) for the absorption coefficient.

$\frac{\partial S L}{\partial f}=f d\left(\frac{2.2 \cdot 10^{-4}}{\left(1+f^{2}\right)^{2}}+\frac{360.8}{\left(4100+f^{2}\right)^{2}}+5.5 \cdot 10^{-7}\right)-\frac{18}{f \ln 10}$

It is obvious that, for any $d$, the limits of $\frac{\partial S L}{\partial f}$ for $f$ tending to 0 and $+\infty$ equal to $-\infty$ and $+\infty$ respectively. Thus, there exists at least one frequency $f^{*}$ where the source level $S L^{*}$ is minimal. We use the same assumption as in [13] with a $3 \mathrm{~dB}$ narrowband around $f^{*}$. Fig. 2 shows the optimal frequency and minimum source level to guarantee a SNR of $25 \mathrm{~dB}$ at the receiver.

The final step is to compute the energy consumption to transmit a packet of $l$ bits at a bitrate $b$ bps. As we are using acoustic waves, the sound intensity of a source is related to a reference intensity and is given by

$$
I_{t}=10^{S L / 10} I_{\text {ref }}
$$

where $I_{r e f}=\frac{p^{2}}{2 \rho c}$ with $p$ is the effective sound pressure, $\rho$ the density of sea water and $c$ the propagation velocity of the sound wave in sea water. For simplicity, we assume a constant speed of $c=1500 \mathrm{~m} / \mathrm{s}$ and we take $I_{\text {ref }}=$ $0.67 \cdot 10^{-18} \mathrm{~W} / \mathrm{m}^{2}$.

In the case of cylindrical spreading, the power $P_{t}$ required to achieve intensity $I_{t}$ at $1 \mathrm{~m}$ from the source in the direction of the receiver is expressed as

$$
P_{t}=2 \pi z I_{t}
$$

TABLE I

COST MATRIX FOR THE SIMPLE GAME (COST OF ROW PLAYER, COST OF COLUMN PLAYER).

\begin{tabular}{c|c|c|c|c|} 
& $\mathrm{CC}$ & $\mathrm{CD}$ & $\mathrm{DC}$ & $\mathrm{DD}$ \\
\hline $\mathrm{CC}$ & $2 c_{1}, 2 c_{1}$ & $2 c_{1}, c_{1}$ & $c_{1}, c_{1}+c_{2}$ & $c_{1}, c_{2}$ \\
\hline $\mathrm{CD}$ & $c_{1}, 2 c_{1}$ & $c_{1}, c_{1}$ & $c_{1}, c_{1}+c_{2}$ & $c_{1}, c_{2}$ \\
\hline $\mathrm{DC}$ & $c_{1}+c_{2}, c_{1}$ & $c_{1}+c_{2}, c_{1}$ & $c_{2}, c_{2}$ & $c_{2}, c_{2}$ \\
\hline $\mathrm{DD}$ & $c_{2}, c_{1}$ & $c_{2}, c_{1}$ & $c_{2}, c_{2}$ & $c_{2}, c_{2}$ \\
\hline
\end{tabular}

where $P_{t}$ in watt and $z$ is the depth in meter.

For commercial hydrophones [9], the energy needed to receive a packet is typically around one fifth of the transmitted energy. Thus, the energies to receive and transmit a packet of $l$ bits at a bitrate $b$ bps are

$$
E_{t}=P_{t} \frac{l}{b} \quad \text { and } \quad E_{r}=\frac{1}{5} E_{t}
$$

In our model, we take a packet size of 128 Bytes and a channel capacity of $20 \mathrm{~kb} / \mathrm{s}$.

\section{Simple Game}

This game is a simplified model of an UWSN and aims at introducing the cooperation issues in an underwater environment. The analysis is split in two. The first part focuses on non-reactive strategies and the second uses evolution theory to propose new reactive strategies.

In this scenario, we define a game where the players are the sensors. In each time slot, a sensor has four possible strategies that are the combination of actions regarding its packet and the opponent's packet. A sensor can either cooperate $(C)$ or defect $(D)$. Thus, the tuples are the followings:

CC means that the player will send his packet to the opponent and forward the opponent's packet to the opponent's buoy.

$C D$ means that the player will send his packet to the opponent and drop the opponent's packet.

$D C$ means that the player will send his packet directly to his buoy and forward the opponent's packet to the opponent's buoy.

$D D$ means that the player will send his packet directly to his buoy and drop the opponent's packet.

A cost is assigned to a certain strategy and is related to the energy consumption of each node. As the energy consumption is mostly dictated by the transmission loss (2), we neglect the noise for simplicity. Thus, the costs of transmission for the two distances is given by

$$
c_{1}=d_{1}^{k} 10^{d_{1} \alpha(f) 10^{-4}} \quad \text { and } \quad c_{2}=d_{2}^{k} 10^{d_{2} \alpha(f) 10^{-4}}
$$

We do not assign a cost when a packet is dropped. We can describe the game with Table I in which we show these costs.

A buoy has a history $H=\left(h_{1}, h_{2}, \ldots, h_{l}\right)$ of length $l$ in which we store the outcome $h=\{1,0\}$ (success '1' or failure ' 0 ') of the previous games. The success rate $w$ of a buoy is the number of received packets over the total sent packets. 
If the number of moves played is smaller than the history length, we assume a success ' 1 ' in the missing history ${ }^{1}$.

The information set is the history and the strategies are a function mapping every possible history to an action. The strategy space is big. For instance, with the history 01011, the actions allowed are $C C, C D, D C$ and $D D$. Thus, for a history length $l$, we have $2^{l}$ possible histories. As a result, we have $4^{2^{l}}$ strategies.

Therefore, we make some restrictions on this space. We propose two kinds of strategies: reactive and non-reactive. The reactive strategies take a decision based on the opponent's last moves and will be analyzed in Section V-B. When the success rate is smaller than a given threshold $\rho$, the sensor cannot choose risky actions $C *$. Therefore, it has to make only safe moves such as $D *$ to successfully transmit the data packet to the buoy. We can see this additional constraint as two states: risky and safe states. Moreover, if the opponent is "dead", the sensor plays $D D$. Non-reactive strategies take a fixed move in the risky state and also a fixed move in the safe state. They are of the form $m / m^{\prime}=* * / D *$ where $m$ and $m^{\prime}$ are the moves chosen in risky and safe states respectively. For instance, $C D / D C$ selects $C D$ in a risky state and $D C$ in a safe state. Hence, we have 8 non-reactive strategies. These strategies are the same two-step strategies defined in [2].

The payoff increases by one at each time slot until the authority has no sensors left. Thus, the payoff is the network lifetime.

\section{A. Non-Reactive Strategies}

For simplicity, we do not use the full communication model but only the cost described previously. The sensors are at the depth $z=600 \mathrm{~m}$. The distances between each sensor are $d_{1}=1000 \mathrm{~m}$ and $d_{2}=1897.4 \mathrm{~m}$. The memory slots vary between 5 and 10. After running an exhaustive search on the strategies space and playing around with the parameters, we obtain a matrix containing the tuple of payoffs for each pair of strategies. With (1) and this matrix, we identify the pairs that are Nash equilibria. We found that, for $\rho<0.6$, there exist four $\mathrm{NE}(C D / D C, C D / D C),(C D / D C, C D / D D)$, $(C D / D D, C D / D C),(C D / D D, C D / D D)$. These NE have all the same lifetime. However, above 0.6, a new Nash equilibrium $(C C / D D, C C / D D)$ appears with the highest lifetime. Interestingly, this Nash equilibrium results in full cooperation, whereas the other fours are full defection. Above 0.8, The four Nash equilibra collapse in $(C D / D D, C D / D D)$ with a lifetime still below $(C C / D D, C C / D D)$.

These results show that cooperation can emerge underwater but with slight differences from terrestrial communications [2]. When the threshold $\rho$ is low, there are 4 uncooperative NE for the underwater scenario instead of one. In addition, $\rho$ has to be high enough to see a cooperative NE. It is not the case in terrestrial settings because the cooperative NE always exists. However, $\rho$ has to be greater than 0.33 to have the highest lifetime.

${ }^{1}$ Simulations tell us that a uniformly distribution of ' 1 ' and ' 0 ' or all ' 1 ' in the missing history are equivalent and lead to the same Nash equilibria.

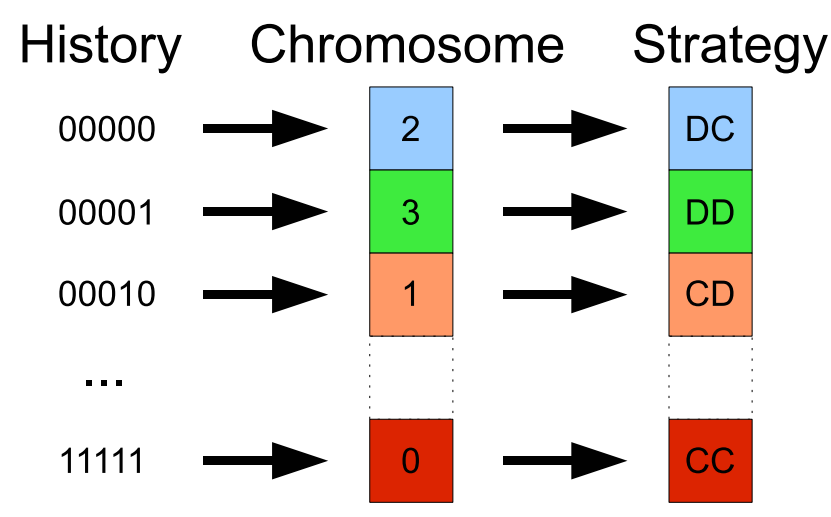

Fig. 3. Genotype encoding: the history, once converted into an integer, represents the locus on the chromosome and the related base encodes the move. For instance, the history 00010 converted to an integer is 2 . At the locus (position) 2 on the chromosome, we find the base 1 which encodes $C D$.

\section{B. Evolving Strategies}

After the analysis of non-reactive strategies, we wonder if there exist better strategies. In the following, we extend the strategy space to take into account reactive strategies based on previous opponent's moves. The idea of this analysis is to take a set of strategies and evolve them according to their performance. This should eventually converge to the best strategies. To do so, we borrow tools from evolutionary biology and use the well-known genetic algorithm (GA) optimization technique.

A GA has a population of candidate solutions (individuals) represented as chromosomes, which evolves toward better solutions. It requires a genetic encoding of solutions and a fitness function to evaluate them. We are interested in the NE leading to the best strategies. Therefore, we would like to evolve the strategies and to do so, we need to encode them. Recall that a reactive strategy is a function mapping a move to every possible history. With a history of 5 , the history 01011 means that the last game was a failure, the next to last game was a success and so on. As there are $2^{5}$ possible histories, the chromosome length is 32 . The history, once converted into an integer, represents the locus and the related base encodes the move $(0=C C, 1=C D, 2=D C$ and $3=D D$ ). We interpret the locus of each base of the chromosome as a decision. Therefore, there are $4^{32}$ possible chromosomes (or strategies). Fig. 3 gives an example of this encoding.

The fitness function measures the quality of a solution and in our model it is the lifetime. Thus, we would like to select strategies that achieve the best lifetime. We now describe, step by step, one iteration of the algorithm. First, we create an initial population by generating randomly 50 individuals. Each individual passes a validation test to see if the bases conform to the corresponding history. Recall that the threshold $\rho$ forces a strategy to be in a safe state and thus reduces the number of possible moves.

After that, each individual plays the iterated game we previously explained against himself and the rest of the 


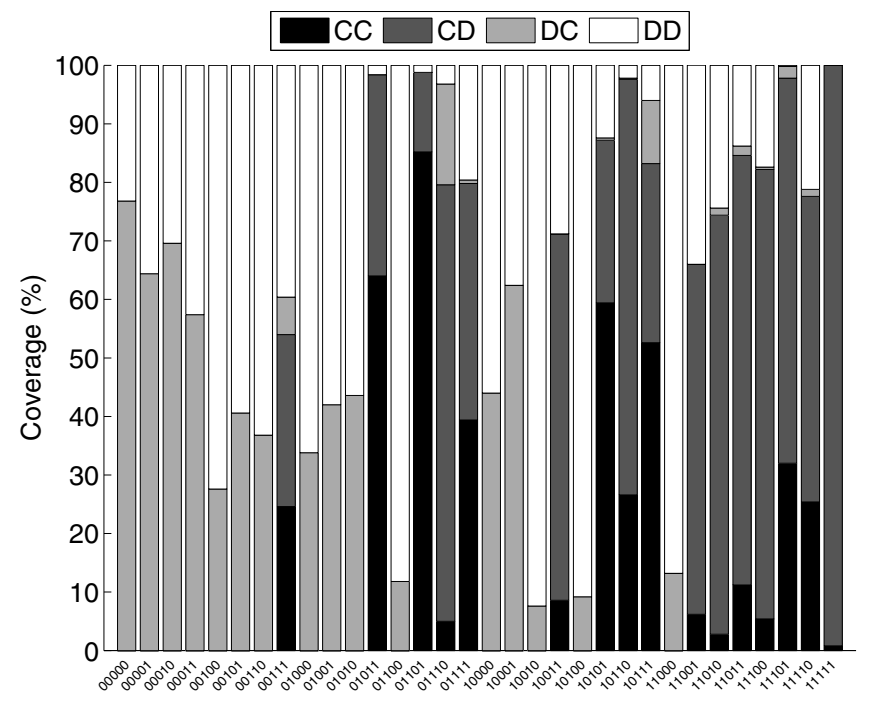

Fig. 4. Representative chromosome for a history of 5: bases coverage of the best strategies. The $\mathrm{x}$-axis represents the locus (history).

population. The parameters of the game remain the same except for the memory slot of 5 and 10 , and $\rho=0.6$. Individuals are selected based on their fitness. We perform an elitist selection where $10 \%$ of the top individuals are picked up to feed the new population. Then, we complete the new population by applying crossover and mutation on the set of selected individuals. With a probability of 0.6 , the crossover simply takes two chromosomes and swaps everything between two points set at random. This leads to two offspring. On a single chromosome, the mutation alters the bases of each locus with a probability of 0.01 . The new population is then used in the next iteration of the algorithm. We stop the algorithm after 1000 generations.

We ran 500 genetic algorithms and analyzed the best strategies. Fig. 4 shows the coverage of the bases for each locus among the 500 best strategies. In other words, the chromosome depicted is the representative chromosome of 500 best strategies. The $\mathrm{x}$-axis represents the locus or history, whereas the $y$-axis is the distribution of the bases or moves.

The reactive and non-reactive strategies we defined previously were pure strategies. However, the coverage graph could be interpreted as a single mixed strategy. If we sort the distribution of bases by their corresponding success rate instead of locus, then we can plot Fig. 5 for 5 and 10 memory slots, which is more meaningful than the full-locus coverage graph. As we can see, the strategy tries to exploit the opponent while the success rate is high enough. When the opponent is not exploitable and the success rate drops, the strategy starts being nicer with full cooperation $(C C)$ but also stops cooperating $(D D)$. When the success rate is below the threshold, only safe moves are allowed and, interestingly, the strategy tends to prefer $D D$ to $D C$.

We compared this strategy to the non-reactive ones and found that the only Nash equilibrium was when the two players use the evolved strategy. Thus, this strategy outperforms the non-reactive ones. The results of both reactive

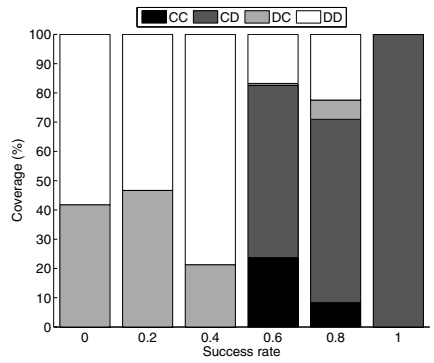

(a) History of 5

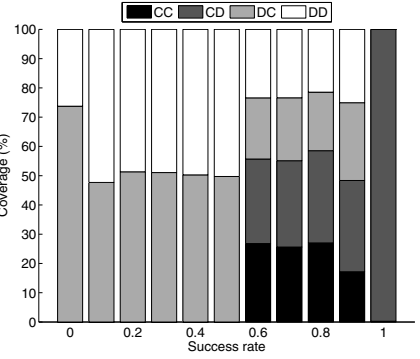

(b) History of 10
Fig. 5. Bases coverage of the best strategies ordered by their success rate.

and non-reactive strategies support our idea that cooperation can emerge underwater and could be, depending on the parameters, the best strategy.

\section{EXTENDED Game}

In this second scenario, we extend now the previous game to study the cooperation of two authorities in more realistic conditions. We will see what influences cooperation and how.

Each buoy keeps a history of the number of received packets over the total number of sent packets for the last 5 time slots. The success rate of this history is defined as the average of the value stored in the history. We limit our analysis to the non-reactive strategies of the previous game. Again, the goal of this game is to maximize the lifetime of each network.

In our simulations, we varied two parameters: the threshold $\rho$ and the number of sensors per authority. We made 500 runs for each parameters and each run had a new topology. We performed an exhaustive search on the strategies space. For each run, we selected the Nash equilibria that are Paretooptimal corresponding to the highest lifetime. Due to the random distribution of the sensors, the topology was different for each run. Therefore, a pair of strategies that was a cooperative $\mathrm{NE}$ in one run, could be an uncooperative one or not even a NE in another run. Hence, we classified each $\mathrm{NE}$ according to its level of cooperation as following:

1) No cooperation: both players forward nothing.

2) Semi-cooperation: one player forwards some of the opponent's packets, the other forwards nothing.

3) Full cooperation: both players forward some of the opponent's packets.

The summary of the parameters for this simulation is in Table II. This extended version uses the communication model described in Section IV.

Fig. 6 and 7 provide a comparison between the two scenarios. The ordinate of each graph shows the percentage of Nash equilibria for each cooperation level over the 500 runs. In Fig. 6, we see the distribution of NE for different thresholds with 50 sensors per authority. In both scenarios, the percentages of Nash equilibria leading to no and semicooperation are almost the same. Full-cooperative NE are less than $10 \%$ for the distinct location scenario and $2 \%$ for shared location scenario. When an authority requests 
TABLE II

PARAMETERS FOR THE SIMULATION.

\begin{tabular}{l|l} 
Parameter & Value \\
\hline \hline Pool & $10 \times 10 \times 1 \mathrm{~km}$ \\
\hline Position of the buoys & $\begin{array}{l}(2.5,5) \mathrm{km} \text { and }(7.5,5) \mathrm{km} \\
\text { or }(5,5) \mathrm{km}\end{array}$ \\
\hline Memory slot & 5 \\
\hline Sensors per authority & $10,20,50,100$ \\
\hline Distribution of sensors & random \\
\hline Battery & $0.001 \mathrm{~J}$ \\
\hline Threshold & $0.7,0.8,0.9$ \\
\hline
\end{tabular}

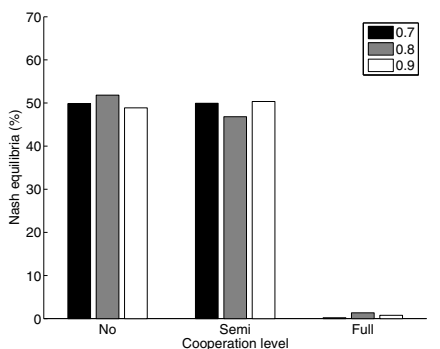

(a) shared location

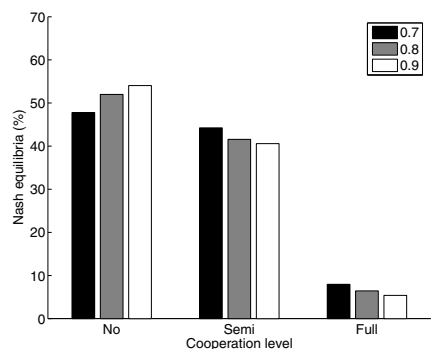

(b) distinct location
Fig. 6. Distribution of Nash equilibria for different thresholds with 50 sensors per authority.

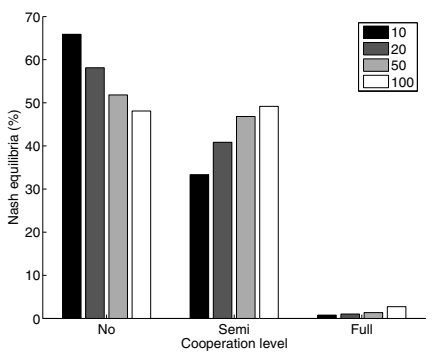

(a) shared location

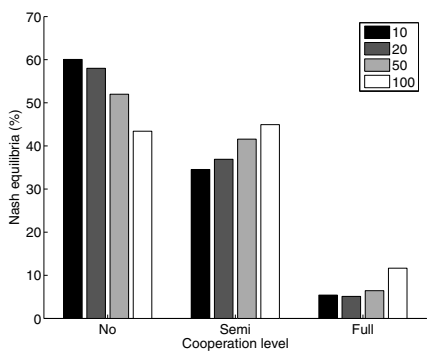

(b) distinct location
Fig. 7. Distribution of Nash equilibria for different number of sensors per authority with $\rho=0.8$.

a higher quality of service and increases its threshold, in the scenario of shared location, the distribution does not change. We distinguish a slight modification for the distinct location scenario, which tends to decrease the semi- and fullcooperative NE for the advantage of non-cooperative NE.

Fig. 7 shows the distribution of Nash equilibria for 10 , 20, 50 and 100 sensors per authority with $\rho=0.8$. As in the previous figure, there are less full-cooperative $\mathrm{NE}$ in the shared location scenario than in the distinct location scenario. However, we observe that when the number of sensors increases, the percentage of semi- and full-cooperative $\mathrm{NE}$ increases as well for both scenarios. Full-cooperative $\mathrm{NE}$ remain lower than semi-cooperative ones. Around 100 sensors and higher, semi-cooperative Nash equilibria exceed non-cooperative NE. The number of sensors alters more significantly the cooperation level than the threshold.

With this game, we have seen what and how coopera-

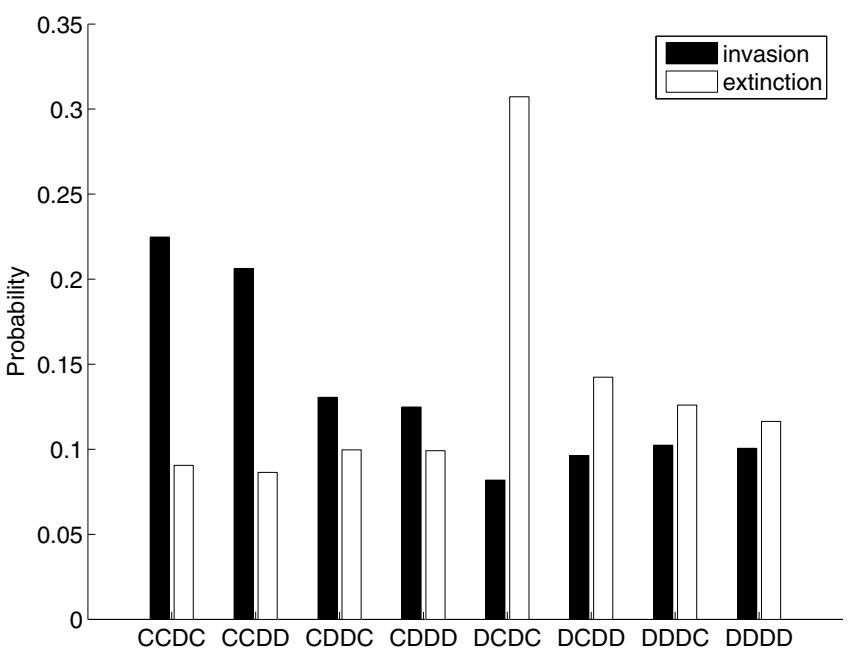

Fig. 8. Probability of invasion and extinction.

tion is influenced. In particular, the strategies but also the topologies and the parameters affect the cooperation level. As predicted with the game in Section $\mathrm{V}$, a form of cooperation can emerge spontaneously without incentives under certain conditions and it consists of at least one network forwarding packets for the other.

\section{Evolutionary GAME}

In this section, we analyze the strategic interactions between three and more authorities in an underwater setting. More specifically, we address the issues of the deployment of underwater sensor networks when one or more are already deployed with known strategies, and we study how the cooperation evolves with the help of evolutionary game theory.

This scenario has 10 authorities. Each authority governs 20 networks with 5 sensors each. The buoys of each networks are located at the center of the pool. The parameters are the same as in Table II except for $\rho=0.8$. The strategy set is the non-reactive strategies of Section V.

The first simulation challenges one strategy against another. The initial population profile is $\vec{x}=(0.8,0.2)$. This means that a small group of mutants plays against the rest of the population. We take the average over 100 topologies with, for each one, 100 runs of the evolution.

Table III shows the percentage of topologies in which mutants have invaded the population. The rows are the initial strategy and the columns are the mutants. For instance, the strategy $C C / D C$ invades at $83.8 \%$ the strategy $D C / D C$ but with only $1.8 \%$ the strategy $D C / D C$ invades $C C / D C$. During the simulation, we observed that, sometimes, the number of generations required to kill off the mutants are very short (around 5 or 10). This usually happens when the current configuration (topology, distribution of strategies) is optimal or near optimal for the initial population. We also saw that a strategy known for being the best for the current topology cannot invade and eventually dies off. This could explain the low percentage in some cases. 
TABLE III

PERCENTAGE OF TOPOLOGIES IN WHICH MUTANTS HAVE INVADED THE ENTIRE POPUlATION. COLUMNS ARE THE MUTANTS.

\begin{tabular}{c||c|c|c|c|c|c|c|c|} 
& $\mathbf{C C / D C}$ & $\mathbf{C C / D D}$ & $\mathbf{C D / D C}$ & $\mathbf{C D / D D}$ & $\mathbf{D C} / \mathbf{D C}$ & $\mathbf{D C} / \mathbf{D D}$ & $\mathbf{D D} / \mathbf{D C}$ & $\mathbf{D D} / \mathbf{D D}$ \\
\hline \hline CC/DC & & 9.3 & 13.0 & 11.3 & 1.8 & 8.2 & 8.9 & 11.0 \\
\hline CC/DD & 7.1 & & 11.6 & 11.5 & 3.0 & 7.3 & 10.8 & 9.1 \\
\hline CD/DC & 10.4 & 11.0 & & 11.5 & 9.2 & 9.0 & 10.0 & 8.7 \\
\hline CD/DD & 10.2 & 9.8 & 11.9 & & 9.0 & 10.6 & 9.3 & 8.6 \\
\hline DC/DC & 83.8 & 70.7 & 14.5 & 13.4 & & 11.3 & 10.3 & 11.1 \\
\hline DC/DD & 19.4 & 18.3 & 13.9 & 13.8 & 11.7 & & 11.3 & 11.2 \\
\hline DD/DC & 15.4 & 12.7 & 13.6 & 13.0 & 11.7 & 10.9 & & 10.8 \\
\hline DD/DD & 11.1 & 12.6 & 12.8 & 12.8 & 10.8 & 10.2 & 11.0 & \\
\hline
\end{tabular}

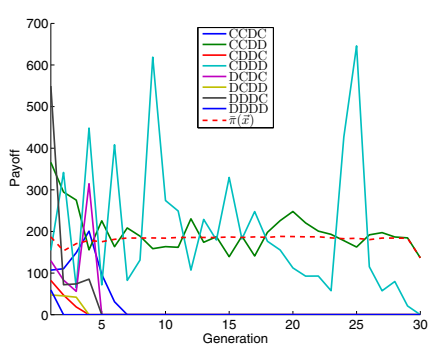

(a) payoff

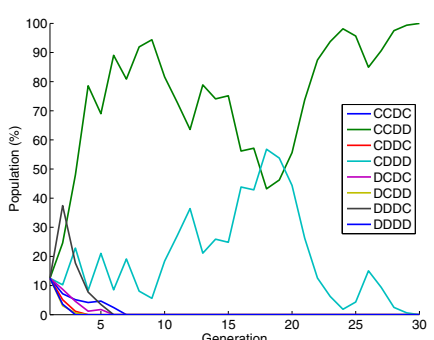

(b) population

Fig. 9. An example of evolution.

With Table III, we can plot Fig. 8 which represents the probability of invasion and extinction of each strategy against the others. We differentiate three classes of invading strategies. The first is the strategies that do not seek cooperation like $D C / D C$ or $D D / D D$ with a probability of invasion below 0.1 and a probability of extinction above 0.1. $D C / D C$ has a probability of extinction higher than the average because it seems to be the less efficient. The second class of strategies is those that exploit the others and do not cooperate $(C D / D C$ and $C D / D D)$. They have a probability of invasion around 0.125 and a probability of extinction around 0.1 . The last class and maybe the most interesting is $C C / D C$ and $C C / D D$ with a probability of invasion higher than 0.2 and a probability of extinction lower than 0.1 . These strategies seek cooperation and cooperate regarding opponents' packets.

The last simulation aims at identifying evolutionary stable strategies among all strategies. To do so, we distribute uniformly the 8 strategies to the population. The initial population profile is then $\vec{x}=$ $(0.125,0.125,0.125,0.125,0.125,0.125$,

$0.125,0.125)$ and the parameters for the simulations are the same as previously. Fig. 9 shows an example of the evolution for the payoffs and the population profile. The oscillations are due to the random distribution of strategies among the networks.

The probability of invasion per strategy is depicted in Fig. 10. The two highest probabilities, respectively $C C / D D$ and $C D / D D$, correspond exactly to the two Nash equilibria found in Section V for $\rho=0.8$. Between these two NE, we found that $C C / D D$ had the highest lifetime and we observe here that it has also the highest probability of invasion.

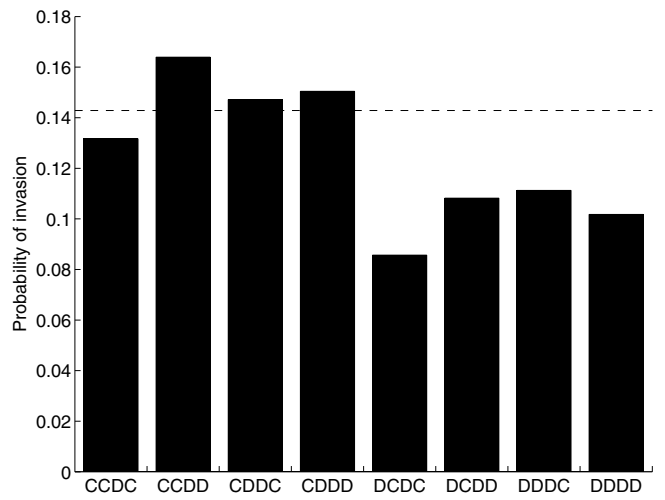

Fig. 10. Probability of invasion. The dashed line is the average.

We cannot conclude that these two strategies are strictly ESS because sometimes they die off due to the topology. We refer to them however as weak ESS. A weak ESS is a strategy evolutionary stable for some topologies but that has a probability of invasion at least higher than the average.

In at least $80 \%$ of the cases, a strategy cannot invade. Therefore, when deploying an underwater sensor network, the new authority should take into consideration that the others might not change their strategies. Thus, the new authority can take advantage of this and fine tune its strategy accordingly.

\section{CONCLUSION}

First, we have introduced, using a simple game, the issues of cooperation in an underwater environment. We analyze two kinds of strategy: non-reactive and reactive. For the former, the Nash equilibria lead to either full defection or full cooperation under certain conditions. To have full cooperation, the quality of service has to be high enough. We evolve a set of reactive strategies to find the best one and observe that it outperform the non-reactive ones. These initial analyses show that cooperation can emerge underwater.

With realistic conditions, we have shown that the strategies, but also the topologies and the parameters, affect the cooperation level. As a result, a form of cooperation can emerge without incentives under certain conditions and consists of at least one authority forwarding packets for the other.

We have studied in the last game the evolution of strategies when a new UWSN is deployed, while two or more are 
already running. We have seen that Nash equilibria of the first simple game are also weak evolutionary stable strategies. Because the probability of invasion is low, a new authority wanting to deploy an UWSN should take into account that the other authorities might not change their strategies. Thus, the new authority can take advantage of this to exploit the others.

In future work, we could investigate cooperative schemes for medium access control, which take into account multipath propagation. Dynamic topologies with mobile nodes could also be considered as the water current is not negligible. Finally, we could focus future studies on cooperative models of network flows with players of different interests.

\section{REFERENCES}

[1] K. Akkaya and M. F. Younis. A survey on routing protocols for wireless sensor networks. Ad Hoc Networks, 3(3):325-349, 2005.

[2] L. Buttyan, T. Holczer, and P. Schaffer. Spontaneous cooperation in multi-domain sensor networks. In ESAS 05, 2005.

[3] L. Buttyán and J.-P. Hubaux. Stimulating cooperation in selforganizing mobile ad hoc networks. Mob. Netw. Appl., 2003.

[4] L. Buttyan and J.-P. Hubaux. Security and Cooperation in Wireless Networks. Cambridge University Press, 2007.

[5] G. V. Crosby and N. Pissinou. Evolution of cooperation in multi-class wireless sensor networks. In $L C N$ 07, 2007.

[6] M. Felegyhazi, J.-P. Hubaux, and L. Buttyan. Cooperative packet forwarding in multi-domain sensor networks. PerCom 2005, 2005.

[7] S. Ganeriwal and M. B. Srivastava. Reputation-based framework for high integrity sensor networks. In SASN 04, 2004.

[8] C. Haiguang, W. Huafeng, Z. Xi, and G. Chuanshan. Reputation-based trust in wireless sensor networks. MUE 07, 2007.

[9] LinkQuest Inc. Underwater acoustic modem. http://www.linkquest.com, 2007.

[10] M. J. Osborne. An Introduction to Game Theory. Oxford University Press, 2003.

[11] J. Partan, J. Kurose, and B. N. Levine. A survey of practical issues in underwater networks. In WUWNet 06, 2006.

[12] E. Rasmusen. Games and Information: An Introduction to Game Theory. Blackwell Publishers, 4th edition, 2006.

[13] M. Stojanovic. On the relationship between capacity and distance in an underwater acoustic communication channel. In WUWNet 06, 2006.

[14] W. H. Thorp. Analytic description of the low-frequency attenuation coefficient. Journal of Acoustical Society of America, 1967.

[15] R. Urick. Principles of underwater sound. McGraw-Hill, 3rd edition, 1983.

[16] J. von Neumann and O. Morgenstern. Theory of Games and Economic Behavior. Princeton University Press, 1944.

[17] J. N. Webb. Game Theory: Decisions, Interaction and Evolution. Springer, 2007.

[18] S. Zhong, J. Chen, and Y. R. Yang. Sprite: a simple, cheat-proof, credit-based system for mobile ad-hoc networks. INFOCOM 03, 2003. 